\title{
SOBRE METODOLOGÍA DE GRAMÁTICA HISTÓRICA DEL ESPAÑOL: A PROPÓSITO DE UNA OBRA RECIENTE
}

\author{
ÁNGEL LóPEZ GARCía \\ Universidad de Valencia \\ angel.lopez@uv.es
}

\begin{abstract}
Resumen
Contributions to Spanish historical morphology are used to dealing with many descriptive issues, but they generally lack methodological considerations. This was not the case with other components of historical grammar such as the phonological and the lexical ones. However morphology, perhaps because of its semiproductive character and the irregularity of its paradigms, has been conceived of as a sample of unrelated data. This paper aims to consider the paradigmatic proposals that have been recently made by Joel Rini putting them in the methodological context of current research on grammaticalization in general linguistics.
\end{abstract}

\section{Sobre una carencia de la morfología histórica del español}

Hace cuatro años se publicó un libro notable que no ha merecido, a mi entender, el reconocimiento que hubiera sido de esperar: el de Joel Rini (1999). Se me podría decir que esta obra ha tenido buenas reseñas, que, de vez en cuando, es citada en los congresos y que, en general, no ha pasado desapercibida. Así es, en efecto, pues apareció en una editorial muy. conocida y los filólogos han podido manejarlo sin problemas. Pero a lo que quiero referirme es a su dimensión metodológica, aspecto de cuya trascendencia tal vez ni el propio autor sea plenamente consciente.

Se trata de un libro inusual en el panorama bibliográfico de la Morfología histórica del español. Desde que la Filología románica fuera fundada por los Diez y Meyer-Lübcke y desde que Ramón Menéndez Pidal la aplicara -magistralmente, con todas las reservas que se quiera, esa es la verdad- al español, lo cierto es que la Lingüística ha experimentado cambios de una magnitud mareante, y así se ha modificado también nuestra visión de los hechos de forma paralela. De los hechos fónicos: después de las revisiones de la escuela de Praga y de la llamada Fonología natural, ya nadie acepta el ingenuo mecanicismo fisiológico de las leyes de los neogramáticos y la aportación -más asombrosa conforme va pasando el tiempo- de Emilio Alarcos ha acabado por imponerse. De los hechos sintácticos: todos somos conscientes de que la sintaxis pidaliana, como la de los demás neogramáticos, era vestigial y de que la visión funcional (o sea, pragmático-cultural) que introdujeron Rafael Lapesa y su escuela es el único camino posible. De los hechos léxicos: aunque no con el mismo grado de extensión que los anteriores componentes, también el 
léxico lo vemos ahora en su evolución de otra manera, visión a la que no son ajenas las decisivas contribuciones del diccionario histórico de Joan Corominas y José Antonio Pascual o del diccionario del español medieval de Bodo Müller, así como los planteamientos estructurales de Eugenio Coseriu y de sus discípulos (Salvador, Geckeler, etc.). Pero en este panorama relativamente confortable hay una carencia: la de la descripción de los hechos morfológicos, los cuales a menudo se siguen enfocando, mutatis mutandis, como en tiempos de Menéndez Pidal.

Existen dos razones, que se alimentan mutuamente, las cuales explican este hecho tan sorprendente. La primera, a mi entender, es la irregularidad del componente morfológico. La moderna Lingüística se caracteriza por ser una disciplina cognitiva que se basa en la existencia de pautas de regularidad, de reglas y de patrones: el predominio de aquellas caracteriza al generativismo; el de estos, al funcionalismo; pero la preferencia por la visión sistemática de los hechos es común a ambas orientaciones. El retraso en la consideración estructural de los fenómenos morfológicos no es tan sólo una peculiaridad de la morfología histórica del español: se da también en la morfología sincrónica y en todo tipo de lenguas. En segundo lugar, la Lingüística interna ha ido constituyéndose como ciencia de manera progresiva, desde los componentes con menor número de unidades hasta los más complejos: la Fonología se desarrolla en los años veinte y treinta del pasado siglo; la Morfología, en los cuarenta y cincuenta; la Sintaxis es la ciencia lingüística de los sesenta y setenta; en los ochenta, asume un sesgo semántico; en los noventa, se torna pragmática y discursiva. En el pensamiento occidental, la Lingüística se ha desarrollado paralela y simultáneamente a ambos lados del Atlántico. Naturalmente, la fonología de Trubetzkoy no es la de Swadesh o Twaddell ni la sintaxis chomskiana es la de Danes, pero, aun así, resulta fácil establecer las semejanzas entre ellas. Salvo en el caso de la morfología, una vez más. La segunda guerra mundial fue la responsable de que, mientras en EEUU se desarrollaba una profunda renovación conceptual y metodológica de los estudios morfológicos, animada por los Bloomfield, los Hockett, los Wells o los Harris, Europa, con alguna honrosa excepción como Hjelmslev, permanecía muda. Ello determinó que, mientras el estudio de la morfología histórica del español continuaba vigoroso en América de la mano de Yakov Malkiel y Steven Dworkin, se interrumpiera en Europa, con la consiguiente sacralización del legado pidaliano. Sólo una obra escrita en colaboración como la de $\mathrm{M}$. Alvar y B. Pottier (1983) venía a introducir un tímido sesgo cognitivo -imputable a B. Pottier- y sociolingüístico -responsabilidad de M. Alvar- en fecha tan tardia como 1983: en cualquier caso no fue suficiente para modificar un status quaestionis esclerotizado como el que todavía padecemos.

\section{Comprender no es sólo almacenar}

Sirva este largo preámbulo para situar el libro que reseñamos en su justo contexto. Debo advertir ya de entrada que la aportación de Joel Rini me parece una contribución valiosa al conocimiento de la morfología histórica del español. Pero, sobre todo, me parece una contribución relevante, lo que no es lo mismo. Me explicaré. J.R. habría podido limitarse a recoger sus contribuciones anteriores en relación con la materia (sobre la $-y$ de hay o sobre /ée/>/éi/, por ejemplo) y añadir otras nuevas (sobre ove > hube y casos similares; sobre el extraño paradigma de dormir y morir; sobre veer > ver y sus análogos; sobre eres, etc.). $\mathrm{Y}$, 
en efecto, lo hace, reuniendo así un conjunto de propuestas interesantes, aunque discutibles, sobre algunos problemas que el lector avisado reconoce entre los más obstinados caballos de batalla de la morfología histórica hispánica. Pero J. R. hace algo más: después de señalar acertadamente lo inapropiado de los planteamientos metodológicos que le han precedido, encabeza su libro con un capítulo de consideraciones teóricas. Esto es algo que cualquier libro de Lingüística, como cualquier trabajo científico en general, debería dar por supuesto. Pero el hecho es que en este dominio no ha sido así y de ahí la singularidad del libro de Rini. Esta preocupación teórica y metodológica (al fin y al cabo la obra no ha aparecido en una serie dedicada al español, sino en una colección titulada Current Issues in Linguistic Theory) se manifiesta a lo largo de todas sus líneas y me parece lo fundamental de su trabajo, hasta el punto de convertirlo en un punto de inflexión de la disciplina.

Es a comentar este aspecto y sus repercusiones concretas a lo que dedicaré las líneas que siguen. Hace poco se ha publicado una reseña filológica muy positiva sobre el libro de Rini (Thibault, 2002). Sin embargo, en mi opinión, da una impresión engañosa del mismo $y$, para los intereses de algunos lectores, contiene un mensaje desmotivador de su lectura. $\mathrm{Y}$ es que, aparte de una exposición detallada de su contenido, esta reseña constituye una buena muestra de las limitaciones del método tradicional. Evidentemente, casi todas las observaciones del reseñista son acertadas, su erudición es notable y su entusiasmo ante el masivo aporte de datos del trabajo de Rini -logrado gracias al uso del archivo digital ADMYTE fundamentalmente- resulta contagioso. Pero uno no puede evitar disentir de Thibault cuando liquida en cuatro líneas la referencia al primer capítulo del libro de J.R. diciendo que éste critica la terminología tradicional sobre algunos conceptos metodológicos y que «lo que más parece molestarle al autor» es que se use el término analogía para referir a situaciones de nivelación paradigmática.

\section{Metodología, no terminología}

Dudo que se pueda estar de acuerdo con un planteamiento así. La innovación de Rini no es terminológica (no se limita a llamar a las cosas de otra manera), es metodológica. Lo que ha hecho es postular un método diferente y, al hacerlo, nos obliga a ver el mundo de la morfología medieval del español de otra manera. Es verdad, dicho sea en descargo de Thibault, que a veces parece que se habla de una simple manera de redenominar las cosas, como cuando J. R. escribe:

Rather, analogical change is but one type of morphological change. Moreover, there will be no need to use the terms "proportional analogy" or "four-part analogy" since analogical change in the present view [las cursivas son mías] necessarily involves at least four parts presented in the form of a proportion (14).

Pero junto a term está view y esto es lo decisivo. Por eso, cuando J. R. resume su aportación de este primer capítulo metodológico lo hace como sigue:

As discussed in Chapter 1, leveling is one of the two most systematic types [la cursiva es mía] of morphological change (the other being analogy), yet specialists of Spanish historical grammar have not dealt with this phenomenon in any sort of systematic way (29). 
O sea que lo que se propone J. R. es ejemplificar el alcance de los dos procesos sistemáticos que operan en la evolución morfológica, la analogía y la nivelación (junto con otros que representan variaciones suyas).

¿Se trata de una aportación trivial? J. R. afirma lo siguiente:

By no means do I wish to imply, however, that what follows is the only way to conceptualize morphological change. Nor is the following conceptualization intended to be a "new theory" of morphological change.

Pues bien, contra lo que el propio autor afirma, yo me permito dudarlo. La teoría no está expuesta como tal, cierto. Pero el libro constituye una muestra clarísima de que todos los problemas que trata pueden resolverse bien como procesos analógicos, bien como procesos de nivelación. Y esto es muy significativo. Por ejemplo, sería niveladora la extraña evolución de dormir y morir, frente a cubrir, cumplir, fundir, subir, etc., los cuales unifican la vocal temática por nivelación a su vez: el mantenimiento de la $o$ de dormir, morir viene inducido por la primera persona duermo, muero, cuya ue se trata como o sobre el modelo puedo /poder, frente a la $u$ de cubro, cumplo, fundo, subo. En cambio, es analógica la retroformación de rey, ley sobre el plural reyes, leyes a base de la proporción 'marqués es a marqueses como rey a reyes': no se puede aceptar la simple evolución fonética rege > *ree $>$ rei, pues pe(d)em no dio *pey sino pie.

La diferencia teórica entre la analogía y la nivelación estriba en que la primera es el resultado de una proporción realizada desde un modelo, mientras que la segunda afecta a todo un paradigma: dada la semejanza de aver y tener, el modelo de ove induce la nueva forma tove. Como dice Rini a propósito de la analogía:

This type of morphological change necessarily involves two base forms and one derived form [el modelo] ... The two base forms are connected ... by a semantic, syntactic, or structural (i.e., morphological) similarity. Based on base form 1 and its derived form, and through the connection ... betweem base form 1 and base form 2 , a new form is produced (12).

En cambio:

Leveling differs greatly from analogical change in that it does not involve two different base forms (and their respective derived forms) ... Rather, it occurs in a well-defined parameter called a "paradigm", i.e., the set of inflected forms of a given word. These inflected forms, by their very nature, are already connected by semantic, syntactic, and in most cases, morphological similarity (14).

\section{La teoría de la gramaticalización}

Hasta aquí la aportación de Rini. Sin embargo, quisiera destacar que las implicaciones derivadas de reducir la evolución morfológica, en lo esencial, a la analogía y a la nivelación son muy profundas. Rini no llega a extraerlas porque no es su trabajo, él es un filólogo -con alcances generalistas, eso sí-, pero no un teórico del lenguaje. Mas, puestos a examinar la cuestión como lingüistas generales, resulta inevitable que pasemos del descubrimiento del método al de la teoría, del cómo al porqué. Si las formas del español (y de las demás len- 
guas) han evolucionado como lo han hecho, por analogía o por nivelación, es probablemente porque se trata de los dos patrones formales que pueden regir cualquier evolución de formas. $\mathrm{Y}$ en este punto es irremediable que adoptemos una perspectiva mucho más abstracta, la de la teoría morfodinámica general, que es una rama de las Matemáticas. Réné Thom (1990) ha definido dos conceptos que intervienen en cualquier proceso de producción de formas, ya sea en la naturaleza, ya en el cerebro humano ( $y$, por lo tanto, en el lenguaje): la pregnancia y la saliencia. Las saliencias son formas individuadas; las pregnancias son acciones propagativas emitidas por las formas salientes (se dice que las pregnancias las catectizan) y que recaen en otras formas salientes induciendo en ellas determinadas transformaciones formales que se conocen como efectos figurativos.

La pregnancia tiene una entidad ontológica distinta de la saliencia, de forma que la alcanza o es emitida por ella, pero no podría ponerse en su lugar. En realidad, para Thom estos conceptos, sobre todo el de pregnancia, tienen un claro valor dinámico. Aunque da una definición muy general de ambos, es conveniente captar estos conceptos por sus ejemplos. Supongamos que una corriente fluida, que es una pregnancia, llega hasta un obstáculo, hasta una piedra situada en el centro de su cauce; cuando la alcance, la piedra, que es una forma con saliencia, opuesta al avance del fluido, será desplazada parcialmente y, tal vez, erosionada de manera característica, pero, en cualquier caso, también modificará la corriente pregnante convirtiéndola en una segunda pregnancia con turbulencias. La definición que da Thom (1992: 14) de ambos conceptos es, por supuesto, formalista:

Ces expériences fondamentales de notre perception nous obligent à considérer deux types d'entités: les objets saillants dans notre champ perceptif (qui se manifestent à l'Ego par des discontinuités du temps interne), et d'autre part ces entités à caractère irréversiblement rayonnant, qui suscitent chez les formes saillantes des aspects "perceptifs" eventueliement variables. Ceci m'a conduit à introduire à coté des formes matérielles saillantes, ces entités ubiquistes qui suscitent des changements d'aspect chez les formes saillantes qu'elles investissent: j'ai proposé d'appeler "prégnances" (objectives) ces entités ... Toute prégnance est émise à partir d'objets sources (saillants); dans sa propagation une prégnance investit certaines formes saillantes, c'est à dire produit en elle des transformations d'ètat ayant des effets observables (effets figuratifs). La forme saillante investie par une prégnance (p) peut réémettre cette prégnance sous une forme diférente ( $\left.p^{\prime}\right)$. La transformation $(p)>\left(p^{\prime}\right)$ peut alors apparaitre -dans certains cas- comme un "codage".

Pues bien, adviértase que lo que Rini llama analogía tiene todo el aspecto de funcionar siguiendo las pautas de las saliencias y lo que llama nivelación se ajusta a los patrones de las pregnancias. El modelo de la relación analógica, lo que J. R. llama la forma derivada, es como un saliente que modificará la vinculación semántica establecida entre dos o más bases (es decir, una pregnancia preexistente) induciendo una o más formas nuevas que al relacionarse entre sí darán lugar a una nueva corriente pregnante. En cambio, un paradigma es claramente una pregnancia, un flujo que conduce todas sus partículas en la misma dirección y que tiene fuerza suficiente para asimilar y llevarse consigo a las partículas opuestas a la marcha general del movimiento: en esto consiste la nivelación.

El funcionamiento de la sucesión encadenada de saliencias y pregnancias, de analogía, operada sobre el modelo de una forma derivada, y de nivelación, que irradia en el marco de todo un paradigma, se aprecia en el tratamiento que propone J. R. para la evolución veer > 
ver. En principio y por razones estrictamente fonéticas, habria sido de esperar que vée se hubiese transformado en vé antes que veémos en vémos, pero los datos estadísticos de la Primera Crónica procedentes de ADMYTE no avalan esta conclusión, sino la prolongada alternancia de ambos tipos en todas las personas. Ello conduce al autor a sustituir la explicación fonética por la morfológica. Primero hubo una fusión de las vocales en hiato en el infinitivo, inducida por las secuencias de futuro y condicional en las que la vocal acentuada del auxiliar hacía difícil mantener el hiato en el auxiliado: veér + há / hia = vér + há / hía. A continuación, esta forma vér actúa de modelo para un proceso analógico: temér es a temémos, podér a podémos, etc., como vér es a vémos. Finalmente, vémos ( $\mathrm{y}$ védes) extienden por nivelación la supresión del hiato a todo el paradigma haciendo vées $>$ vés, vée $>$ vé, etc.

Obsérvese que lo que tenemos es una sucesión "nivelación > analogía > nivelación". La ausencia, en el tema, de hiato entre vocales similares resulta ser general en el paradigma verbal (poder, podemos), esto es, existe un proceso nivelador que se toma como punto de partida. Dicha nivelación previa, que es una corriente pregnante, tropieza con una situación anómala, con una saliencia, la de veer. La pregnancia modifica la saliencia, convierte veer en ver allí donde la resistencia de la forma saliente es más débil, en el futuro y en el condicional. Pero la saliencia así modificada se convierte en seguida en fuente de una nueva nivelación, por lo que ver induce vemos que lleva a ve, etc.

En conclusión, diremos que este libro resulta de lectura inexcusable para especialistas en morfología histórica del español, pero también para lingüistas generales. Al proponer dos únicos grandes procesos de cambio morfológico, la analogía y la nivelación, está sentando las bases de un acercamiento entre la Morfología y la Sintaxis históricas, disciplinas que desde que se planteó la teoría de la gramaticalización por P. Hopper y E. Traugott (1993) parecen haber funcionado como compartimentos estancos. Aunque sólo fuera por ello, y con independencia de sus valiosas contribuciones al conocimiento del español medieval, el esfuerzo ya habría merecido la pena.

\section{Referencias bibliográficas}

Alvar, M. y B. Pottier, (1983): Morfología histórica del español, Madrid, Gredos. Hopper, P. y E. Traugott (1993): Grammaticalization, Cambridge University Press.

Rini, J. (1999): Exploring the Role of Morphology in the Evolution of Spanish, Amsterdam/ Philadelphia, John Benjamins.

Thibault, A. (2002): "Reseña a J. Rini, Exploring the Role of Morphology in the Evolution of Spanish", R.Li.Ro., vol. 66, págs. $270-276$.

Thom, R. (1990): Esbozo de una Semiofisica, Barcelona, Gedisa.

Thom, R. (1992): Pouvoirs de la forme, Aarhus, Aarhus Universitet. 